\title{
BMJ Open Neighbourhood deprivation and lung cancer risk: a nested case-control study in the USA
}

Maureen Sanderson, ${ }^{1}$ Melinda C Aldrich, ${ }^{2}$ Robert S Levine, ${ }^{3}$ Barbara Kilbourne, ${ }^{4}$ Qiuyin Cai, ${ }^{5}$ William J Blot ${ }^{5}$

To cite: Sanderson M, Aldrich MC, Levine RS, et al. Neighbourhood deprivation and lung cancer risk: a nested case-control study in the USA. BMJ Open 2018;8:e021059. doi:10.1136/ bmjopen-2017-021059

- Prepublication history and additional material for this paper are available online. To view these files, please visit the journal online (http://dx.doi. org/10.1136/bmjopen-2017021059).

Received 8 December 2017

Revised 21 May 2018

Accepted 7 August 2018

Check for updates

(C) Author(s) (or their employer(s)) 2018. Re-use permitted under CC BY-NC. No commercial re-use. See rights and permissions. Published by BMJ.

${ }^{1}$ Department of Family and Community Medicine, Meharry Medical College, Nashville, Tennessee, USA

${ }^{2}$ Department of Thoracic Surgery, Vanderbilt University Medical Center, Nashville,

Tennessee, USA

${ }^{3}$ Department of Family and Community Medicine, Baylor College of Medicine, Houston, Texas, USA

${ }^{4}$ Department of Sociology, Tennessee State University, Nashville, Tennessee, USA ${ }^{5}$ Division of Epidemiology, Department of Medicine, Vanderbilt University School of Medicine, Nashville, Tennessee, USA

Correspondence to Dr Maureen Sanderson; msanderson@mmc.edu

\section{ABSTRACT}

Objectives To examine the association between neighbourhood deprivation and lung cancer risk. Design Nested case-control study.

Setting Southern Community Cohort Study of persons residing in 12 states in the southeastern USA.

Participants 1334 cases of lung cancer and 5315 controls.

Primary outcome measure Risk of lung cancer.

Results After adjustment for smoking status and other confounders, and additional adjustment for individual-level measures of socioeconomic status (SES), there was no monotonic increase in risk with worsening deprivation score overall or within sex and race groups. There was an increase among current and shorter term former smokers $(p=0.04)$ but not among never and longer term former smokers. There was evidence of statistically significant interaction by sex among whites, but not blacks, in which the effect of worsening deprivation on lung cancer existed in males but not in females.

Conclusions Area-level measures of SES were associated with lung cancer risk in current and shorter term former smokers only in this population.

\section{INTRODUCTION}

Cigarette smoking is the predominant cause of lung cancer, but additional environmental factors may contribute to risk and be amenable to detection via examination of geographic patterns of cancer in relation to neighbourhood economic indices. Although lung cancer rates have decreased over time in the USA, from 2010 to 2014, the age-adjusted incidence rate was 55.8 per 100000 , and the comparable death rate was 44.7 per 100000 . $^{1}$ Our prior work identified lung cancer mortality hot spot maps within US counties from the 1980 s to $2009 .^{23}$ The greatest concentration occurred across southeastern states and varied by sex and race. These distinct geographic patterns of lung cancer mortality may be indicating different exposures and/or causal pathways. Analyses of county-level data showed positive associations between lung cancer mortality and smoking,

\section{Strengths and limitations of this study}

Large size and diversity of cohort under observation enhancing our ability to detect trends.

- Adjustment for individual-level smoking status using several dimensions of smoking exposure history.

- Use of the Nam-Powers-Boyd occupational status score as a measure of individual-level socioeconomic status.

- Neighbourhood deprivation index was developed at the census tract level rather than the block group level, but smaller block groups should be more homogenous and span a wider range of values.

- Inability to completely rule out residual confounding by smoking suggesting caution in interpretation of the study findings.

poverty, coronary heart disease mortality and National Air Toxics Assessment excess cancer deaths. ${ }^{4}$

The present analysis focuses on poverty that may be present at the individual level and/or at the area level. Krieger et a $\check{\bar{l}}$ postulated that area-level socioeconomic status (SES) may be a more comprehensive measure of SES than individual-level SES, because it captures social characteristics of communities that are not typically measured. A meta-analysis of 64 studies of individual-level measures of socioeconomic position (SEP) and lung cancer incidence reported significantly increased risk for lowest versus highest educational SEP (relative risk (RR) 1.61, 95\% CI 1.40 to 1.85), occupational SEP (RR 1.48, 95\% CI 1.34 to 1.65 ) and income-based SEP (RR $1.37,95 \%$ CI 1.06 to 1.77$).{ }^{6}$ Few studies have examined lung cancer incidence associated with area-level measures of SES alone ${ }^{78}$ or in combination with individual-level measures of SES, ${ }^{9-11}$ and only one of these studies has been conducted in the USA. ${ }^{12}$

We performed a multilevel nested casecontrol study of lung cancer incidence within the prospective Southern Community Cohort Study (SCCS) to examine the association 
between area-level neighbourhood deprivation together with smoking and other individual-level risk factors. We hypothesised that area-level socioeconomic deprivation would be positively associated with lung cancer, independently of smoking, and other individual-level risk factors. In addition, we hypothesised this positive association would persist after control for individual-level measures of SES. Given our prior findings for lung cancer mortality, ${ }^{23}$ we also hypothesised that these associations would differ by sex and race. Lastly, we hypothesised that these associations would differ by smoking status since lung cancer death is markedly lower 10 years after quitting, ${ }^{13}$ and the lung cancer screening guidelines use a cut-off of 15 years since quitting smoking. ${ }^{14}$

\section{METHODS}

Detailed methods of the SCCS ${ }^{15}$ appear elsewhere. Briefly, the SCCS is comprised of 9842 white men and 15463 white women, 22905 black men and 32457 black women, and 1566 men of other races and 2564 women of other races in 12 southeastern states aged $40-79$ years who completed a baseline questionnaire between 2002 and 2009. Men and women were primarily low-income with the majority $(86 \%)$ enrolled in person at community health centres and the remaining men and women randomly sampled from the general population. Depending on the enrolment site, in-person interviews or mailed questionnaires were completed. After enrolment, 1395 persons with incident lung cancer were identified through linkage with state cancer registries and the National Death Index. Information on lung cancer histology (squamous cell carcinoma, adenocarcinoma, small cell carcinoma, large cell carcinoma and other) was collected from the cancer registries. Approximately four controls were chosen by incidence density sampling and matched to each case on sex, race, age at diagnosis $( \pm 2$ years increased to \pm 5 years if necessary), enrolment source (community health centre and general population) and year of recruitment for a total of 5580 controls. Controls were not matched to cases on recruitment site to avoid overmatching on residence. Exclusions included races other than white or black, and unknown neighbourhood deprivation index resulting in 1334 cases and 5315 controls for analysis.

In this multilevel nested case-control study, baseline surveys collected information on individual-level lung cancer risk factors including smoking status, total exposure to secondhand smoke, chronic obstructive pulmonary disease (COPD) and SES. Smoking status was categorised as never, former and current; within former was further stratified as time since last smoked, and current was categorised using number of cigarettes smoked per day as characterised by Blot $e t a l .{ }^{16}$ The number of hours participants reported they were around the cigarette smoke of other people at home and at other indoor places including work was totaled and categorised as none and using tertiles among controls reporting more than none. COPD was identified using International Classification of Diseases, Ninth Rivision, Clinical Modification (ICD9) codes from Medicaid or Medicare reports ${ }^{17}$ and using self-report for the 1877 (28.2\%) of cases and controls who were not enrolled in Medicaid or Medicare. Our decision to use claims data for the majority of participants was based on the finding of substantial under-reporting of COPD among those with claims data. Individual-level measures of SES included annual household income, education level, health insurance coverage and the Nam-Powers-Boyd (NPB) occupational status score, ${ }^{18}$ which was defined using quartiles among controls. The NPB occupational status score was developed from the 2000 US Census as a measure of SES of occupations and ranged from 1 to 100 reflecting the lowest to highest socioeconomic standing. The score was based on responses to a question about the longest job held during adulthood in which we scored each occupation and used the average score within 20 distinct categories.

A multistage approach that included automated and interactive processes was used to maximise success of geocoding SCCS participants' addresses at baseline and follow-up. ${ }^{19}$ A total of $99.96 \%$ of participant addresses have been geocoded with only 5.2\% at the ZIP code centroid level (2.8\% post office box and $2.3 \%$ non-post office box addresses). SCCS staff used Federal Information Processing Standard codes for geocoded participant addresses to link individual-level SCCS data with area-level data on deprivation detailed below. Information on neighbourhood deprivation was updated with each follow-up. However, participants' residence at study baseline was used for area-level data since nearly $65 \%$ of persons had identical neighbourhood deprivation index levels during follow-up. Values for the $35 \%$ of participants who had different neighbourhood deprivation index values at baseline and follow-up were somewhat more likely to become more deprived than less deprived ( $\mathrm{n}=861$ more deprived, $\mathrm{n}=610$ less deprived). However, of those who became more deprived, there was no difference between cases and controls (cases $\mathrm{n}=115$, mean change 0.99, SD 0.82 ; controls $\mathrm{n}=746$, mean change 1.00 , SD 0.86 ).

Information from the 2000 Census $^{20}$ for geocoded addresses was determined by spatial join to TIGER/ Line Shapefiles ${ }^{21}$ using ESRI ArcMap software (ESRI, Redlands, California, USA). Using formulas developed by Messer $e t a l^{22}$ and principal components analysis, Signorello et $a t^{23}$ developed a neighbourhood deprivation index at the census tract level for SCCS participants comprising the following constructs: education, employment, occupation, housing and poverty. The 11 characteristics chosen from the Census to represent neighbourhood SES included: percentage of persons that did not graduate high school (age $\geq 25$ years), percentage of males and females who are unemployed, percentage of males in professional occupations, percentage of housing units with $\geq 1$ occupant per room, percentage of occupied housing units with renter/owner costs $>50 \%$ of income, percentage of persons with income below the 1999 poverty status, percentage of female headed households 
with dependent children, percentage of households with income $<\$ 30000$ per year, percentage of households with public assistance income, percentage of households with no car and median household value. Increasing values of neighbourhood deprivation index reflect increasing levels of deprivation. We used neighbourhood deprivation index at the block group level rather than the census tract level as used by Signorello $e t a l^{23}$ as has been recommended by Krieger et al. ${ }^{5}$ Our index ranged from -2.29 to 5.88 and was modelled as quartiles among controls overall and within sex and race since there was no evidence of linearity based on likelihood ratio tests and cubic splines.

Statistical analyses were performed in SAS V.9.4. Conditional logistic regression was used to estimate the OR and $95 \%$ CI of lung cancer associated with area-level neighbourhood deprivation while accounting for effect modification and adjusting for confounding. ${ }^{24}$ Effect modification by sex, race and smoking status was examined by adding product terms of the dichotomous exposure (upper two vs lower two quartiles among controls) and dichotomous putative effect modifiers to logistic regression models and performing likelihood ratio tests $(\mathrm{p}<0.1$ provided evidence of statistically significant interaction). Smoking status was dichotomised as current and former smokers within 15 years since quitting (shorter term former smokers) and never smokers and former smokers who quit more than 15 years ago (longer term former smokers). There was evidence of effect modification of these associations by sex in whites but not in blacks and not by race. Nevertheless, we stratified by sex-race categories (205 white male cases, 492 black male cases, 276 white female cases, 361 black female cases, 817 white male controls, 1958 black male controls, 1097 white female controls and 1443 black female controls) since our findings on lung cancer mortality suggested distinct geographic patterns depending on sex and race. Nor was there evidence of effect modification by smoking status, but we stratified by smoking status since it is strongly related to neighbourhood deprivation and lung cancer risk.

Potential confounders of the association between neighbourhood deprivation and lung cancer included: age, annual household income, number of household members, education level, enrolment source, health insurance coverage, NPB occupational status score, ${ }^{18}$ urban/rural residence defined as metro or nonmetro county using 2003 rural-urban continuum codes, ${ }^{25}$ time at current residence, perceived indoor and outdoor air quality, family history of lung cancer, smoking status (in seven categories), total exposure to secondhand smoke, alcohol intake, COPD and lifetime maximum body mass index (BMI) as categorised in supplementary table 1 . $\chi^{2}$ tests were performed to assess statistically significant (two sided, $\mathrm{p}<0.05$ ) differences between lung cancer cases and controls. Variables were considered confounders if their addition to the models changed the unadjusted OR by $10 \%$ or more. We adjusted for smoking status, COPD and total exposure to secondhand smoke, which met our criteria for model inclusion, and additionally adjusted for annual household income, education level, health insurance coverage and NPB occupational status score as measures of individual-level SES. Tests for trend across quartiles of exposure were performed by entering categorical variables as ordered categorical variables in the model.

We chose not to use multilevel modelling because relatively few participants were clustered within block groups. Of the 4084 block groups in the study, over two-thirds (67.7\%) included one participant $(85.8 \%$ cases, $70.7 \%$ controls), $16.4 \%$ included two participants ( $10.1 \%$ cases, $15.7 \%$ controls), $7.4 \%$ included three participants $(2.2 \%$ cases, $7.2 \%$ controls), $3.8 \%$ included four participants (1.1\% cases, $3.2 \%$ controls) and $4.7 \%$ included five or more participants $(0.8 \%$ cases, $3.2 \%$ controls $)$. Clarke ${ }^{26}$ cautions against the use of multilevel modelling with fewer than five observations per group, which results in unreliable etimates.

\section{Patient and public involvement}

Neither patients nor the public were involved in development of the research question, study design, patient recruitment or study conduct; however, an annual newsletter is sent to study particicipants as a means of disseminating results. ${ }^{27}$

\section{RESULTS}

Online supplementary table 1 presents demographic and other characteristics of lung cancer cases and controls by sex and race. In comparison with controls, lung cancer cases across both sexes and races tended to have the following characteristics: lower household income, lower education level, Medicaid/Medicare, current smokers, exposed to secondhand smoke, COPD and lower maximum BMI. All cases other than white males tended to have a shorter time at current residence and to drink alcohol. White male and female cases tended to have lower NPB occupational status scores and to have a family history of lung cancer. Black male and female cases tended to have an urban residence. White and black female cases tended to have poor or fair indoor and outdoor air quality.

Table 1 presents ORs for the association between quartiles of neighbourhood deprivation index and lung cancer among all cases and controls and stratified by sex and race. After adjustment for smoking status and other confounders, and additional adjustment for annual household income, education level, health insurance coverage and NPB occupational status score, there was no monotonic increase in risk with worsening deprivation score overall or within sex and race groups. Black men in the third quartile of neighbourhood deprivation were at significantly increased risk of lung cancer relative to black men in the lowest quartile before (OR 1.52, 95\% CI 1.08 to 2.14) and after (OR 1.49, 95\% CI 1.05 to 2.12) adjustment 
Table 1 OR for lung cancer associated with neighbourhood deprivation overall and stratified by sex and race, Southern Community Cohort Study, 2002-2009

\begin{tabular}{|c|c|c|c|c|c|c|}
\hline Characteristic & Cases N & Controls N & $\mathrm{OR}^{*}(95 \% \mathrm{Cl})$ & P for interaction & OR† (95\% Cl) & P for interaction \\
\hline & \multicolumn{6}{|c|}{ Overall } \\
\hline \multicolumn{7}{|l|}{ Deprivation } \\
\hline Quartile 1 & 272 & 1329 & 1.00 (referent) & & 1.00 (referent) & \\
\hline Quartile 2 & 342 & 1329 & 1.08 (0.87 to 1.35$)$ & & 1.08 (0.86 to 1.35$)$ & \\
\hline Quartile 3 & 314 & 1330 & 0.98 (0.78 to 1.23$)$ & & 0.93 (0.73 to 1.18$)$ & \\
\hline Quartile 4 & 406 & 1327 & 1.25 (0.99 to 1.58$)$ & & 1.16 (0.91 to 1.47$)$ & \\
\hline $\mathrm{P}$ for trend & & & 0.09 & & 0.39 & \\
\hline
\end{tabular}

White males

$\begin{array}{cllll}\text { Deprivation } & & & & \\ \text { Quartile 1 } & 37 & 206 & 1.00 \text { (referent) } & 1.00 \text { (referent) } \\ \text { Quartile 2 } & 38 & 202 & 0.68(0.37 \text { to } 1.27) & 0.65 \text { (0.33 to 1.28) } \\ \text { Quartile 3 } & 66 & 203 & 1.37(0.79 \text { to } 2.38) & 1.43 \text { (0.78 to 2.62) } \\ \text { Quartile 4 } & 64 & 206 & 1.27 \text { (0.73 to 2.20) } & 1.26 \text { (0.67 to 2.38) } \\ \text { P for trend } & & & 0.12 & 0.11\end{array}$

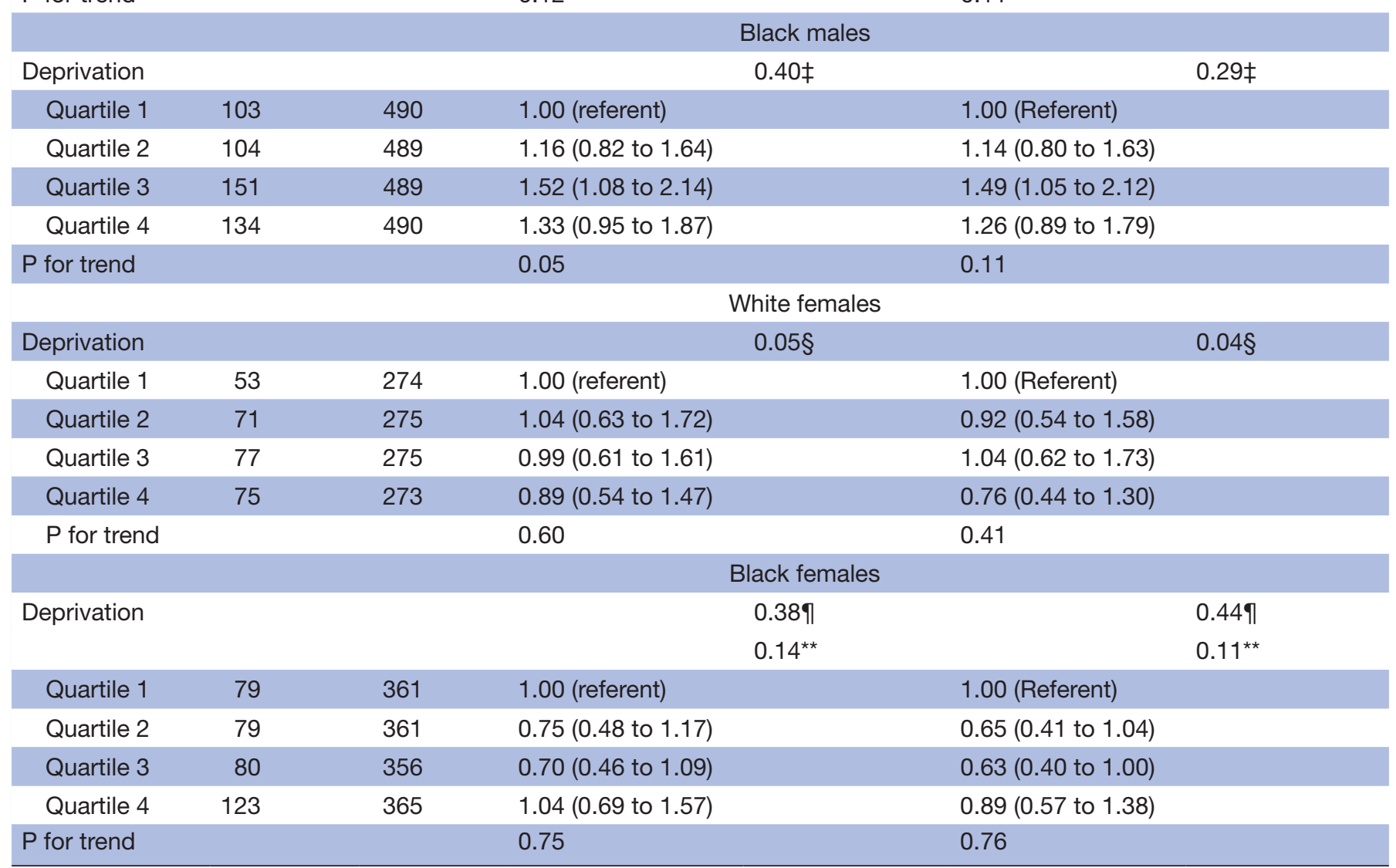

${ }^{*} \mathrm{ORs}$ and $\mathrm{Cl}$ adjusted for smoking status, chronic obstructive pulmonary disease and exposure to secondhand smoke †ORs and $\mathrm{Cl}$ adjusted for smoking status, chronic obstructive pulmonary disease, exposure to secondhand smoke, annual household income, education level, health insurance coverage and NPB occupational status score.

$\ddagger \mathrm{P}$ for interaction comparing white males with black males.

$\S \mathrm{P}$ for interaction comparing white males with white females.

IP for interaction comparing white females with black females.

${ }^{* *} \mathrm{P}$ for interaction comparing black males with black females.

for individual-level measures of SES. There was evidence of statistically significant interaction by sex among whites, but not blacks, in which the effect of worsening deprivation existed in males but not among females.
Table 2 presents ORs for the association between quartiles of neighbourhood deprivation index and lung cancer stratified by smoking status. After adjustment for smoking status and other confounders, but prior to adjustment 
Table 2 OR for lung cancer associated with neighbourhood deprivation stratified by smoking status at baseline, Southern Community Cohort Study, 2002-2009

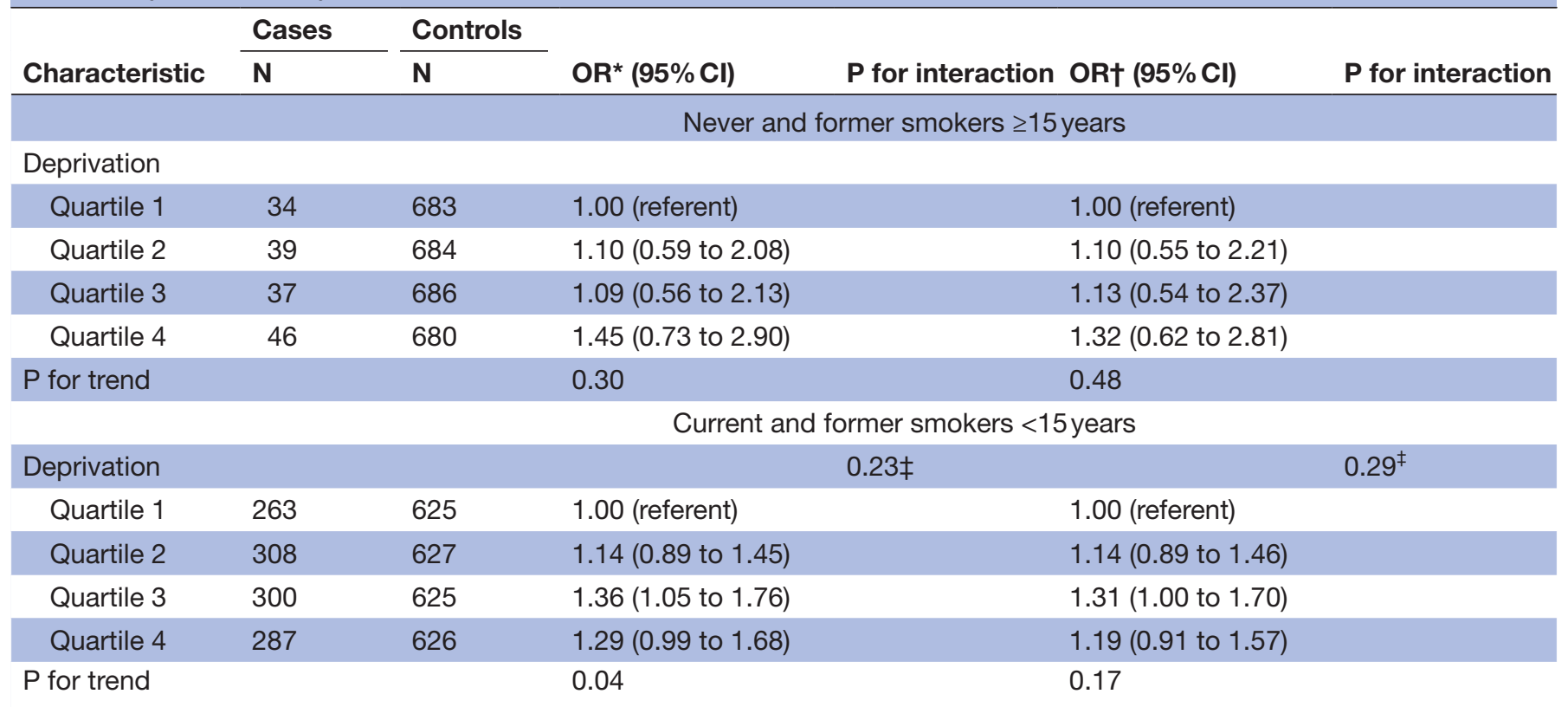

${ }^{*} \mathrm{OR}$ and $\mathrm{Cl}$ adjusted for chronic obstructive pulmonary disease and exposure to secondhand smoke.

†OR and $\mathrm{Cl}$ adjusted for chronic obstructive pulmonary disease, exposure to secondhand smoke, annual household income, education level, health insurance coverage and NPB occupational status score.

$\ddagger P$ for interaction comparing never and former smokers $\geq 15$ years with current and former smokers $<15$ years.

NPB, Nam-Powers-Boyd.

for annual household income, education level, health insurance coverage and NPB occupational status score, there was a monotonic increase in risk with worsening deprivation score among current and short-term former smokers $(\mathrm{p}=0.04)$ but not among never and longer term former smokers. Current and short-term former smokers in the third quartile of neighbourhood deprivation were at significantly increased risk of lung cancer relative to black men in the lowest quartile before (OR 1.36, 95\% CI 1.05 to 1.76 ) adjustment for individual-level measures of SES. There was no evidence of effect modification by smoking status with or without adjustment for individual-level measures of SES.

\section{DISCUSSION}

We found no significantly increased risk of lung cancer associated with greatest neighbourhood deprivation after tight control of smoking overall or within sex and race groups, with a modest attenuation in the association after further adjustment for individual-level SES measures. Similar analyses performed in a more heterogeneous population may differ since the individual-level SES of our population was fairly homogeneous. Most participants reported an annual household income of less than $\$ 25000$ (60.0\% white males, $80.3 \%$ black males, $74.5 \%$ white females and $81.3 \%$ black females) (supplementary table 1). However, a previous analysis of the SCCS found similar positive associations between neighbourhood deprivation and death due to cardiovascular disease, non-malignant diseases and cancer, while positive associations for individual-level SES were restricted to death from cardiovascular disease and non-malignant diseases. ${ }^{23}$ The authors postulated there may be "neighborhood environmental influences on cancer risk above those associated with low SES'.

The somewhat elevated lung cancer risk among those living in the most deprived neighbourhoods was only seen in men, with a significant interaction by sex in whites but not in blacks. A recent review of 36 studies reported that the impact of individual and area level SES on all health outcomes except heart disease was greater for men than for women. ${ }^{28}$ Geronimus $e t a l^{29}$ studied telomere length as a measure of biological ageing due to stress and found that poor whites had shorter telomeres than non-poor whites, which was not the case for blacks or Mexicans. They surmised that some whites may be more affected by socioeconomic difficulties. In a study of earlystage non-small cell lung cancer conducted in Georgia among patients with higher neighbourhood deprivation, blacks were more likely to die but whites were not, even after controlling for surgery. ${ }^{30}$ These authors posited that blacks and whites may experience area-level poverty differently, resulting in different impacts on adverse health outcomes. Our cross-sectional observations might therefore have been influenced by differences in cohort effects which, in turn, could have reflected initial levels rather than magnitudes of deprivation change according to sex and race. 
There was an increased trend of lung cancer among current and shorter term formers smokers that no longer existed after additional adjustment for individual-level measures of SES. Other studies have tended to find increased lung cancer risks associated with lower arealevel SES, some without adjustment for smoking, ${ }^{8912}$ and others with weakened associations after smoking adjustment. ${ }^{71011}$ While the approximate $20 \%-30 \%$ increases in risk we find are small in comparison with the $1000 \%$ or greater increased risks we and others find among cigarette smokers ${ }^{16}$ these increased risks may be signalling the existence of neighborhood-related environmental exposures, social factors and/or behaviours correlated with where one lives that influence lung cancer risk.

Elevated age-adjusted lung cancer mortality rates have been observed in the southern USA for nearly five decades, with the excesses first becoming apparent in seaboard areas where ship manufacturing was common during World War II and then with high rates spreading across inland areas as smoking prevalence became higher in southern states than elsewhere in the USA. ${ }^{2331}$ Much of the excess risk of lung cancer is attributable to the region's higher prevalence of smoking, which has continued to the present ${ }^{32}$ but contributory roles of other environmental factors are possible. The SCCS provided the opportunity to examine associations between social deprivation, as measured by socioeconomic indices recorded by the US Census Bureau for small area neighbourhoods, and individual-level education, income and occupational status, with risk of incident lung cancer. The SCCS population was particularly appropriate for such an analysis because many cohort participants were recruited from disadvantaged, underserved neighbourhoods where deprivation levels would be high. The study also enabled adjustment for smoking and other potential risk factors ascertained through detailed interviews and questionnaires completed by participants.

A potential limitation of our study is the neighbourhood deprivation index we used was developed at the census tract level rather than at the block group level. ${ }^{23}$ However, use of block group-level neighbourhood deprivation rather than census tract-level neighbourhood deprivation has been recommended by others, ${ }^{5}$ and the smaller block groups should be more homogenous and span a wider range of values enhancing our ability to detect trends. Another limitation is the use of a singlelevel analysis that did not account for random effects and was more prone to type I error even with limited data clustering. ${ }^{26}$ A potentially more important limitation is our inability to completely rule out residual confounding by smoking. Smoking is strongly related to lung cancer risk and to SES. Indeed, within the SCCS, we have found that low SES, measured both at the individual level and the neighborhood level, is associated with increasing smoking prevalence. ${ }^{33}$ While we adjusted for current smoking using cigarettes smoked per day categories, and former smoking by years since quitting, minor perturbations in differences within these categories could impact the small increases in risk we find with the deprivation indices. We did not see clear linear or monotonic trends in rising risk with increasing deprivation overall or when stratified by sex and race, suggesting further caution in interpretation of the study findings. The use of a socioeconomically homogeneous sample may limit its generalisability to other populations. A final limitation was the exclusion of races other than white or black ( 57 cases and 228 controls) whose lung cancer risk profile may differ substantially.

Strengths of our study include the large size and diversity of the cohort under observation, the relatively large numbers of lung cancer cases ascertained, the adjustment for individual-level smoking status using several dimensions of smoking exposure history, use of the NPB occupational status score and a comprehensive neighbourhood deprivation index encompassing education, employment, occupation, housing and poverty. Area-level measures of SES appear to impact lung cancer risk among black males only in this population after control for smoking and individual-level measures of SES. We adjusted for the NPB occupational status score, but the somewhat stronger link for men raises the possibility that occupational factors may be involved, since historically men have been more likely than women to be employed in heavy industries and since several occupational exposures in southern industries have long been recognised as lung carcinogens. ${ }^{34}$ Asbestos exposure has been linked to increased lung cancer, but mesotheliomas, the signal of asbestos-induced cancer, have been rare among cohort members (to date five mesothelioma cases vs over 1300 lung cancers among SCCS participants) suggesting that exposures to this carcinogen are not likely to be widespread. Research in at least one southern state (South Carolina) found that socioeconomic deprivation was associated with elevated lifetime cancer risk by air toxics, ${ }^{35}$ and a national US study identified significant positive associations between socioeconomic deprivation and respiratory and cancer hazardous air pollutants. ${ }^{36}$

Our findings may provide clues to further research of the contribution of area-level SES in lung cancer risk. These analyses address associations between high arealevel deprivation and increased lung cancer risk but do not search for areas with high deprivation and low lung cancer incidence. If such discordant areas exist, they would provide additional evidence that area-level deprivation may be non-deterministic, even if it is a determinant. Identification of discordant areas in future studies could have important implications for public health policy.

Contributors MS and WJB contributed equally to this paper. The study was conceived and designed by all authors. The data were analysed by MS. The first draft of the manuscript was prepared by MS, RSL and WJB, which was edited with input from the writing team (MCA, BK and QC). WJB provided the data, and all authors revised the manuscript critically for important intellectual content. All authors gave final approval of the version to be published and have contributed to the manuscript. MS and WJB are guarantors.

Funding This work was supported by grants from the National Institute on Minority Health and Health Disparities (grant P20MD000516) and the National 
Cancer Institute (NCl, grant U54CA163069). The Southern Community Cohort Study was supported by the NCI (grants R01CA092447 and U01CA202979). MCA was supported by the NCl (grant K07CA172294).

Competing interests None declared.

Patient consent Obtained.

Ethics approval The Institutional Review Boards of Meharry Medical College and Vanderbilt University Medical Center approved this study's protocol.

Provenance and peer review Not commissioned; externally peer reviewed. Data sharing statement No additional data available.

Open access This is an open access article distributed in accordance with the Creative Commons Attribution Non Commercial (CC BY-NC 4.0) license, which permits others to distribute, remix, adapt, build upon this work non-commercially, and license their derivative works on different terms, provided the original work is properly cited, appropriate credit is given, any changes made indicated, and the use is non-commercial. See: http://creativecommons.org/licenses/by-nc/4.0/.

\section{REFERENCES}

1. In: Howlader N, Noone AM, Krapcho M, Miller D, Bishop K, et al. eds. SEER Cancer Statistics Review, 1975-2014, National Cancer Institute. Bethesda Cronin KA, MD, 2017. https://seer.cancer.gov/csr/ 1975 2014/.

2. Devesa SS, Grauman DJ, Blot WJ, et al. Cancer surveillance series: changing geographic patterns of lung cancer mortality in the United States, 1950 through 1994. J Natl Cancer Inst 1999;91:1040-50.

3. Levine RS, Kilbourne K, Robinson P, et al. Hot-spot analysis of malignant neoplasm of the trachea, bronchus and lung in the US. Presented to the 2013 American Public Health Association conference. Boston, MA, 2017. https://apha.confex.com/apha/ $141 \mathrm{am} /$ webprogram/Paper286716.html.

4. Kanarek N, Fitzek B, Su SC, Sc S, et al. County lung cancer mortality: a decision tree model for control and prevention. $J$ Public Health Manag Pract 2008;14:E1-9.

5. Krieger N, Williams DR, Moss NE. Measuring social class in US public health research: concepts, methodologies, and guidelines. Annu Rev Public Health 1997;18:341-78.

6. Sidorchuk A, Agardh EE, Aremu O, et al. Socioeconomic differences in lung cancer incidence: a systematic review and meta-analysis. Cancer Causes Control 2009;20:459-71.

7. Nkosi TM, Parent MÉ, Siemiatycki J, et al. Socioeconomic position and lung cancer risk: how important is the modeling of smoking? Epidemiology 2012;23:377-85.

8. Bryere J, Dejardin O, Launay L, et al. Socioeconomic status and sitespecific cancer incidence, a Bayesian approach in a French Cancer Registries Network study. European Journal of Cancer Prevention 2016:1.

9. Meijer M, Bloomfield K, Engholm G. Neighbourhoods matter too: the association between neighbourhood socioeconomic position, population density and breast, prostate and lung cancer incidence in Denmark between 2004 and 2008. J Epidemiol Community Health 2013;67:6-13.

10. Hystad P, Carpiano RM, Demers PA, et al. Neighbourhood socioeconomic status and individual lung cancer risk: evaluating long-term exposure measures and mediating mechanisms. Soc Sci Med 2013;97:95-103.

11. Li X, Sundquist J, Zöller B, et al. Neighborhood deprivation and lung cancer incidence and mortality: a multilevel analysis from Sweden. $J$ Thorac Oncol 2015;10:256-63.

12. Hastert TA, Beresford SA, Sheppard L, et al. Disparities in cancer incidence and mortality by area-level socioeconomic status: a multilevel analysis. J Epidemiol Community Health 2015;69:168-76.

13. U.S. Department of Health and Human Services. How Tobacco Smoke Causes Disease: The Biology and Behavioral Basis for Smoking-Attributable Disease: A Report of the Surgeon General. Atlanta, GA: U.S. Department of Health and Human Services,
Centers for Disease Control and Prevention, National Center for Chronic Disease Prevention and Health Promotion, Office on Smoking andf Health, 2010.

14. Moyer VA. Screening for lung cancer: u.s. preventive services task force recommendation statement. Ann Intern Med 2014;160:330-8.

15. Signorello LB, Hargreaves MK, Blot WJ. The Southern Community Cohort Study: investigating health disparities. J Health Care Poor Underserved 2010;21(1 Suppl):26-37.

16. Blot WJ, Cohen SS, Aldrich M, et al. Lung cancer risk among smokers of menthol cigarettes. J Natl Cancer Inst 2011;103:810-6.

17. Aldrich MC, Munro HM, Mumma M, et al. Chronic obstructive pulmonary disease and subsequent overall and lung cancer mortality in low-income adults. PLoS One 2015;10:e0121805.

18. Nam CB, Boyd M. Occupational status in 2000 Over a century of census-based measurement. Popul Res Policy Rev 2000:327-58.

19. Sonderman JS, Mumma MT, Cohen SS, et al. A multi-stage approach to maximizing geocoding success in a large populationbased cohort study through automated and interactive processes. Geospat Health 2012;6:273-84.

20. U.S. Census Bureau. Census 2000 Summary File 3 - United States / prepared by the U.S. Census Bureau, 2002.

21. U.S. Census Bureau. 2008 TIGER/Line Shapefiles (machine-readable data files) / prepared by the U.S. Census Bureau, 2008.

22. Messer LC, Laraia BA, Kaufman JS, et al. The development of a standardized neighborhood deprivation index. J Urban Health 2006;83:1041-62.

23. Signorello LB, Cohen SS, Williams DR, et al. Socioeconomic status, race, and mortality: a prospective cohort study. Am J Public Health 2014;104:e98-e107.

24. Dupont WD. Statistical Modeling for Biomedical Researchers A Simple Introduction to the Analysis of Complex Data. 2 edn. Cambridge: Cambridge University Press, 2009:97-155.

25. U.S. Department of Agriculture, 2017. 2003 Rural-urban continuum codes https://www.ers.usda.gov/data-products/rural-urbancontinuum-codes.aspx.

26. Clarke P. When can group level clustering be ignored? Multilevel models versus single-level models with sparse data. $J$ Epidemiol Community Health 2008;62:752-8.

27. Signorello LB, Hargreaves MK, Steinwandel MD, et al. Southern community cohort study: establishing a cohort to investigate health disparities. J Natl Med Assoc 2005;97:972-9.

28. Phillips SP, Hamberg K. Women's relative immunity to the socioeconomic health gradient: artifact or real? Glob Health Action 2015;8:27259.

29. Geronimus AT, Pearson JA, Linnenbringer E, et al. Race-ethnicity, poverty, urban stressors, and telomere length in a detroit communitybased sample. J Health Soc Behav 2015;56:199-224.

30. Johnson AM, Johnson A, Hines RB, et al. The effects of residential segregation and neighborhood characteristics on surgery and survival in patients with early-stage non-small cell lung cancer. Cancer Epidemiol Biomarkers Prev 2016;25:750-8.

31. Mason TJ. Cancer mortality in U.S. counties with plastics and related industries. Environ Health Perspect 1975;11:79-84.

32. Jamal A, King BA, Neff LJ, et al. Current cigarette smoking among adults - United States, 2005-2015. MMWR Morb Mortal Wkly Rep 2016;65:1205-11.

33. Cohen SS, Sonderman JS, Mumma MT, et al. Individual and neighborhood-level socioeconomic characteristics in relation to smoking prevalence among black and white adults in the Southeastern United States: a cross-sectional study. BMC Public Health 2011;11:877.

34. Blot WJ, Fraumeni JF. Geographic patterns of lung cancer: industrial correlations. Am J Epidemiol 1976;103:539-50.

35. Rice LJ, Jiang C, Wilson SM, et al. Use of segregation indices, Townsend Index, and air toxics data to assess lifetime cancer risk disparities in metropolitan Charleston, South Carolina, USA. Int J Environ Res Public Health 2014;11:5510-26.

36. Young GS, Fox MA, Trush M, et al. Differential exposure to hazardous air pollution in the United States: a multilevel analysis of urbanization and neighborhood socioeconomic deprivation. Int J Environ Res Public Health 2012;9:2204-25. 